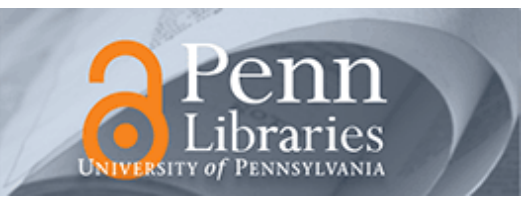

University of Pennsylvania

ScholarlyCommons

\title{
DNA-Decorated Carbon Nanotubes as Sensitive Layer for AIN Contour-Mode Resonant-MEMS Gravimetric Sensor
}

\author{
Chiara Zuniga \\ University of Pennsylvania, zunigac@seas.upenn.edu \\ Matteo Rinaldi \\ University of Pennsylvania, rinaldim@seas.upenn.edu
}

Samuel M. Khamis

University of Pennsylvania, smk@sas.upenn.edu

Timothy S. Jones

University of Pennsylvania, jonests@seas.upenn.edu

Followthis and additional works at: https://repository.upenn.edu/ese_papers

University of Ponncylvania, cjohnson@phyysics.upcnn.edu

\section{Recommended Citation}

Shiara Zuniqa Matteg Rinaldi, Samuel M. Khamis, Timothy S. Jones, A T. Johnson, and Gianluca Piazza, "DNA-Decorated Carbon Nanotubes as Sensitive Layer for AIN Contour-Mode Resonant-MEMS

Gravimetric Sensor", . January 2009.

Copyright 2009 IEEE. Reprinted from:

Zuniga, C.; Rinaldi, M.; Khamis, S.M.; Jones, T.S.; Johnson, A.T.; Piazza, G., "DNA-Decorated Carbon Nanotubes as Sensitive Layer for AIN Contour-Mode Resonant-MEMS Gravimetric Sensor," Micro Electro Mechanical Systems, 2009. MEMS 2009. IEEE 22nd International Conference on , vol., no., pp.320-323, 25-29 Jan. 2009 URL:

http://ieeexplore.ieee.org/stamp/stamp.jsp?arnumber=4805383\&isnumber=4805295

This material is posted here with permission of the IEEE. Such permission of the IEEE does not in any way imply IEEE endorsement of any of the University of Pennsylvania's products or services. Internal or personal use of this material is permitted. However, permission to reprint/republish this material for advertising or promotional purposes or for creating new collective works for resale or redistribution must be obtained from the IEEE by writing to pubs-permissions@ieee.org. By choosing to view this document, you agree to all provisions of the copyright laws protecting it.

This paper is posted at ScholarlyCommons. https://repository.upenn.edu/ese_papers/495

For more information, please contact repository@pobox.upenn.edu. 


\title{
DNA-Decorated Carbon Nanotubes as Sensitive Layer for AIN Contour-Mode Resonant-MEMS Gravimetric Sensor
}

\begin{abstract}
In this work a nano-enabled gravimetric chemical sensor prototype based on single-stranded DNA (ssDNA) decorated single-walled carbon nanotubes (SWNT) as nano-functionalization layer for Aluminun Nitride (AIN) contour-mode resonant-MEMS gravimetric sensors has been demonstrated. Two resonators fabricated on the same silicon chip and operating at different resonance frequencies, 287 and $450 \mathrm{MHz}$, were functionalized with this novel bio-coating layer to experimentally prove the capability of two distinct single strands of DNA bound to SWNT to enhance differently the adsorption of volatile organic compounds such as dinitroluene (DNT, simulant for explosive vapor) and dymethyl-methylphosphonate (DMMP, a simulant for nerve agent sarin). The introduction of this bio-coating layer addresses the major drawbacks of recovery time ( $50 \%$ recovery in less than 29 seconds has been achieved) and lack of selectivity associated with gas sensor based on polymers and pristine carbon nanotube functionalization layers.
\end{abstract}

\section{Keywords}

carbon nanotubes, chemical sensors, density measurement, DNA, carbon nanotubes, contour-mode resonant-MEMS gravimetric sensor, nano-enabled gravimetric chemical sensor prototype, sensitive layer

\section{Comments}

Copyright 2009 IEEE. Reprinted from: Zuniga, C.; Rinaldi, M.; Khamis, S.M.; Jones, T.S.; Johnson, A.T.; Piazza, G., "DNA-Decorated Carbon Nanotubes as Sensitive Layer for AIN Contour-Mode Resonant-MEMS Gravimetric Sensor," Micro Electro Mechanical Systems, 2009. MEMS 2009. IEEE 22nd International Conference on , vol., no., pp.320-323, 25-29 Jan. 2009 URL: http://ieeexplore.ieee.org/stamp/ stamp.jsp?arnumber $=4805383 \&$ isnumber $=4805295$

This material is posted here with permission of the IEEE. Such permission of the IEEE does not in any way imply IEEE endorsement of any of the University of Pennsylvania's products or services. Internal or personal use of this material is permitted. However, permission to reprint/republish this material for advertising or promotional purposes or for creating new collective works for resale or redistribution must be obtained from the IEEE by writing to pubs-permissions@ieee.org. By choosing to view this document, you agree to all provisions of the copyright laws protecting it.

\section{Author(s)}

Chiara Zuniga, Matteo Rinaldi, Samuel M. Khamis, Timothy S. Jones, A T. Johnson, and Gianluca Piazza 


\title{
DNA-DECORATED CARBON NANOTUBES AS SENSITIVE LAYER FOR AIN CONTOUR-MODE RESONANT-MEMS GRAVIMETRIC SENSOR
}

\author{
C. Zuniga ${ }^{1}$, M. Rinaldi ${ }^{1}$, S.M. Khamis ${ }^{2}$, T.S. Jones ${ }^{1}$, A.T. Johnson ${ }^{2}$ and G. Piazza ${ }^{1}$ \\ ${ }^{1}$ Department of Electrical and System Engineering \\ ${ }^{2}$ Department of Physics and Astronomy \\ University of Pennsylvania, Philadelphia, PA.
}

\begin{abstract}
In this work a nano-enabled gravimetric chemical sensor prototype based on single-stranded DNA (ss-DNA) decorated single-walled carbon nanotubes (SWNT) as nano-functionalization layer for Aluminun Nitride (AlN) contour-mode resonant-MEMS gravimetric sensors has been demonstrated. Two resonators fabricated on the same silicon chip and operating at different resonance frequencies, 287 and $450 \mathrm{MHz}$, were functionalized with this novel bio-coating layer to experimentally prove the capability of two distinct single strands of DNA bound to SWNT to enhance differently the adsorption of volatile organic compounds such as dinitroluene (DNT, simulant for explosive vapor) and dymethyl-methylphosphonate (DMMP, a simulant for nerve agent sarin).

The introduction of this bio-coating layer addresses the major drawbacks of recovery time $(50 \%$ recovery in less than 29 seconds has been achieved) and lack of selectivity associated with gas sensor based on polymers and pristine carbon nanotube functionalization layers.
\end{abstract}

\section{INTRODUCTION}

The development of miniaturized smart sensors for real time detection of chemical and biological species has recently been a research topic of primary interest due to the growing number of applications ranging from national safety to medical diagnostics and industrial emission monitoring.

The implementation of high performance gravimetric gas sensors for detection of multiple Volatile Organic Chemicals (VOCs) requires both a high quality transducer and an excellent chemically active adsorbent layer.

Among the wide variety of transducers suggested for chemical sensing, gravimetric sensors based on piezoelectric resonators represent an established category suitable for a broad range of analytes including less polar chemical vapors that generally cannot be effectively sensed by conductance based sensors [1].

MEMS/NEMS devices show great potential as gravimetric sensors since their reduced size and frequency of operation higher than conventional quartz crystal microbalance (QCM) permit them to achieve unprecedented value of mass sensitivity and limit of detection. Although promising, there are still some unsolved challenges related to their effective employment as VOC sensors. For instance, nanocantilever beam based sensors suffer from the greatly reduced size of the area dedicated to the adsorbent layer, which limits the overall mass that can be adsorbed in the presence of a given analyte concentration in the environment. Thin film bulk acoustic resonators (FBAR) can attain high values of sensitivity to adsorbate concentration thanks to their high working frequency and wider area dedicated to sensing, but they cannot provide for different frequencies of operation on the same die, therefore posing a limitation in in the realization of a micromechanical nose with extended sensitivity range.

In this perspective, the AIN MEMS resonant transducer of this work (Fig.1) represents a combination of the key advantages previously demonstrated in MEMS/NEMS technologies. In fact, the AlN resonators have shown very high experimentally-verified sensitivity [2], enhanced effective area dedicated to sensing and the ability to achieve a very wide dynamic range (up to 8 orders of magnitude in sensitivity) by having multiple frequencies on the same chip.

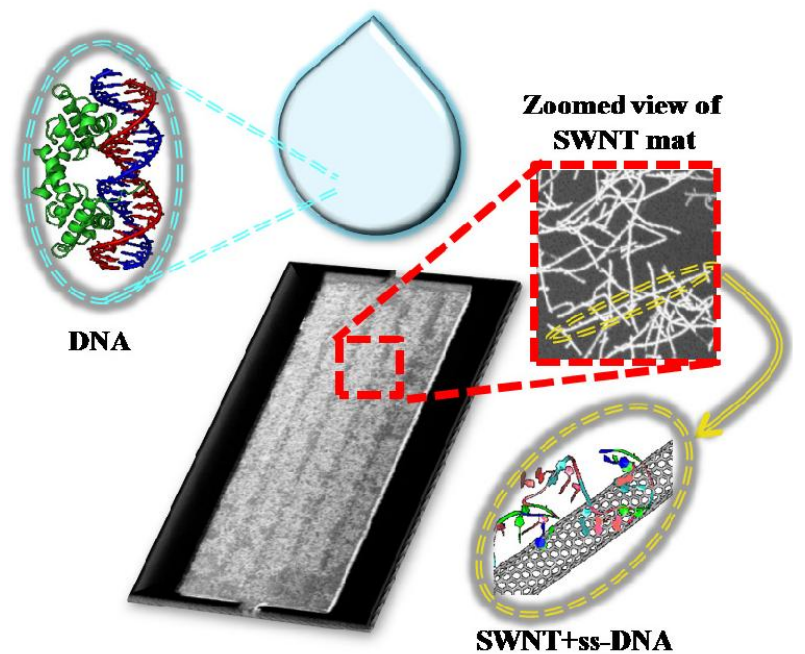

Figure 1: SEM of SWNTs grown on AlN contour mode resonator and schematic representation of the nanofunctionalization process of the adsorbent layer.

In regards to the adsorbent layer, a wide range of nanoengineered materials have been extensively studied and employed [1]. Among those, Single Wall Carbon Nanotubes (SWNTs) stand out as prime candidates for sensing applications because their peculiar hollow structure and large surface to volume ratio greatly enhances their adsorptive capacity. In particular, SWNT bundles offer a great advantage over polymeric films, since they provide the transducer with a larger number of adsorbing sites distributed along two dimensions and therefore enable a quick and reversible adsorption process that solely involves the device surface. In general, this cannot be achieved with polymers, for which the adsorption process occurs within the bulk of the film and is consequently associated with a slower response time.

Generally, the use of bare carbon nanotubes as nanosensitive layer is not sufficient to address the problem of selectivity, since SWNTs are very sensitive to a broad target of molecules. In order to overcome the lack of specificity posed by pristine SWNTs, covalent and 
non-covalent functionalization layers have been employed. SWNTs have been coated by different layers such as chemo-selective polymers [3], enzyme [4], and metal nanoclusters [5]. Although promising, most of these techniques cannot be applied on a large scale and do not allow functionalizing adjacent transducers on the same chip with different chemical coatings. These factors pose an essential limitation to the realization of an on-chip electronic nose.

A novel nano-functionalizing bio-coating constituted by single stranded DNA (ss-DNA) has been successfully demonstrated for SWNT-based FET devices [6]. This technology sets a very promising pathway for the deployment of electronic noses, but it has never been investigated for gravimetric sensor.

In this paper, in order to overcome the common limitations of gas sensor based on pristine CNTs, DNAdecorated SWNT are employed as adsorbent layer for AlN countor-mode resonant sensors. Two resonators fabricated on the same die and operating at different resonance frequencies, 287 and $450 \mathrm{MHz}$, were functionalized with this novel bio-coating layer consisting of SWNTs grown by CVD and two distinct sequences of ss-DNA. Such sensors were tested for gas species such as dinitroluene, DNT, a simulant for explosive vapor and dymethyl-methylphosphonate, DMMP, a simulant for nerve agent sarin, demonstrating a net enhancement in the adsorption of both chemical agents. The analytes employed in this work responded differently to the same strand leading up to an 8 fold enhancement in DNT detection using one sequence of ss-DNA and a 2-3 times increase in both species detection when a second sequence was used.

\section{DESIGN DETAILS AND FABRICATION Sensor Transduction Mechanism}

In an AlN contour-mode resonator, where $W, L$ and $T$ are respectively width, length and thickness of each subdevice (Fig. 2), the application of an electric field across the film thickness produces an in plane dilatation through the equivalent $d_{31}$ coefficient and excites the resonator into lateral contour-extensional vibrations. The frequency of operation is approximately set by the width of a single sub-resonator, $W$ :

$$
f_{0}=\frac{1}{2 W} \sqrt{\frac{E_{0}}{\rho_{0}}}
$$

where $E_{0}$ and $\rho_{0}$ represents the Young's modulus and the density of the resonator.

As previously demonstrated [2], the resonator is best operated as a sensor and attains highest sensitivity when the adsorption layer is placed on its top surface. The large area available renders the resonator extremely sensitive to analyte concentration according to the following equation:

$$
S_{e f f}=\frac{-f_{0}^{2}}{\sqrt{E_{0} \cdot \rho_{0}}} \cdot \frac{W}{T} \quad\left[\mathrm{kHz} \cdot \mu \mathrm{m}^{2} / \mathrm{fg}\right]
$$

where $S_{\text {eff }}$ is the effective sensitivity to analyte concentration of a higher order contour mode resonator. For this kind of piezoelectric transducers the $W / T$ ratio plays a key role, since it is generally greater than 1 and clearly advantageously improves the device sensitivity.

In this work, two devices with frequencies of operation of $287 \mathrm{MHz}(T=2 \mu \mathrm{m}, W=15 \mu \mathrm{m}, L=200 \mu \mathrm{m}$,

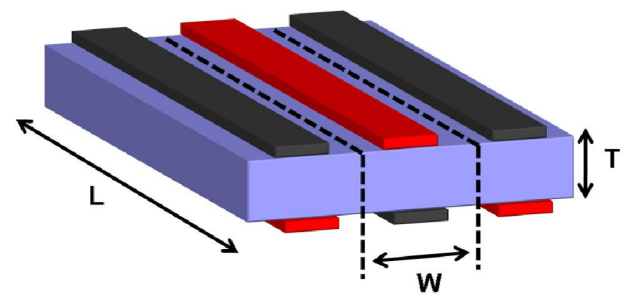

Figure 2: Basic geometry of a higher order laterally vibrating AlN resonator. In this case the number of subresonators, $n$, is 3 .

$n=5)$ and $450 \mathrm{MHz}(T=2 \mu \mathrm{m}, W=10 \mu \mathrm{m}, L=200 \mu \mathrm{m}$, $n=5$ ) were fabricated on the same chip and tested by exposing them to different concentration of analytes. The devices have experimentally confirmed sensitivities to analyte concentration of 17.1 and $27.4 \mathrm{kHz} \cdot \mu \mathrm{m}^{2} / \mathrm{fg}$, which are in close agreement with analytically derived values $\left(17.3 \mathrm{kHz} \cdot \mu \mathrm{m}^{2} / \mathrm{fg}\right.$ and $28 \mathrm{kHz} \cdot \mu \mathrm{m}^{2} / \mathrm{fg}$, respectively). With a device frequency accuracy of $1 \mathrm{ppm}$ (ultimately affected by device $Q$ ) a minimum mass density of $16 \mathrm{ag} / \mu^{2}$ can be resolved by these devices.

Furthermore, recent experimental work [8] on AlN contour-mode resonators has demonstrated devices operating at $7.23 \mathrm{GHz}$ with $Q$ of 630 , proving that even higher sensitivities and Figure of Merit (FOM $=f Q=$ $4 \cdot 10^{12}$ ) can be achieved.

\section{Fabrication}

The fabrication process employed to realize the nanoenabled sensor essentially consists in the combination of top-down microfabrication techniques for the resonator [9] and bottom-up growth of SWNTs by catalytic Chemical Vapor Deposition (CVD) as presented in Figure 2 .

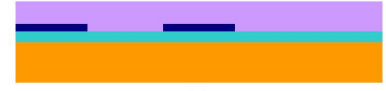

(a)

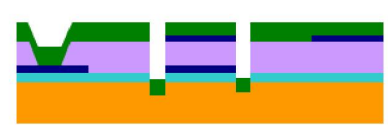

(c)

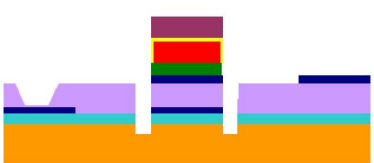

(e)

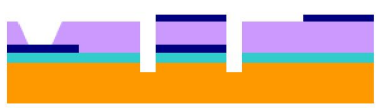

(b)

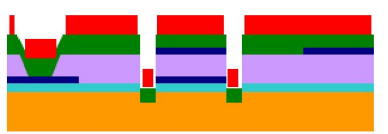

(d)

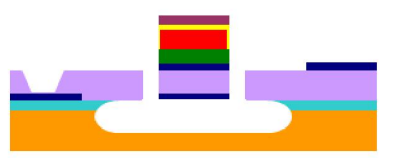

(f)

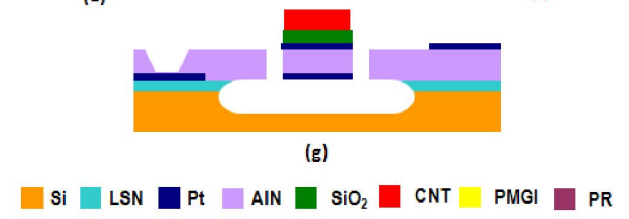

Figure 3: Fabrication process: a) sputter deposition of Pt bottom electrode and AIN on Si and LSN; b) open via in AlN, lithographic patterning of sputtered Pt as top electrode, dry etching of $\mathrm{AlN}$ in $\mathrm{Cl}_{2}$ based chemestry; c) 
sputter deposition of oxide and evaporation of Iron catalyst; d) SWNTS growth by catalytic CVD at $900^{\circ} \mathrm{C}$ in methane containing atmosphere; e) spinning of $P M G I+$ photoresist, development and dry etching of $\mathrm{SiO}_{2}$ in $\mathrm{CF}_{4}$ based chemestry; f) $\mathrm{XeF}_{2}$ dry release of AlN resonator; $g$ ) $P M G I+$ photoresist removal.

The direct integration of SWNTs was performed at the die level immediately before the release of the device from the $\mathrm{Si}$ substrate by $\mathrm{XeF}_{2}$ dry etching. PMGI patterned by standard lithographic techniques protected the body of the resonator during the oxide etching in $\mathrm{CF}_{4}$ chemistry. This step removed the oxide seed layer and SWNTs from every other region of the die but the device body.

The process of integration described above has been performed at the die level because the dimension of the available CVD reactor cannot accommodate a 4-inch wafer substrate, but it can be easily extended to large scale manufacturing [7].

The functionalization of the adsorbent layer represents the last step of the process and it took place after the device fabrication. The single stranded DNA sequences chosen as functionalizing agents were:

\section{Seq. 1: 5' GAC TCT GTG GAG GAG GTA GTC 3' \\ Seq.2: $5^{\prime}$ CTT CTG TCT TGA TGT TTG TCA AAC $3^{\prime}$}

and were applied respectively to the $450 \mathrm{MHz}$ and $287 \mathrm{MHz}$ resonators. The functionalizing agents were obtained from Invitrogen (Carlsbad, CA) and diluted in distilled water to make a solution of $100 \mu \mathrm{M}$. The DNA solution was applied on the device with a micropipette for 45 minutes in a humid environment and hence dried in a nitrogen stream (Fig. 1, 3).

\section{EXPERIMENTAL RESULTS}

The sensors were exposed to varying concentrations of DNT and DMMP before and after the ss-DNA functionalization of the SWNTs adsorbent layer.

Mass flow controllers regulated the flow of high purity argon carrier gas through two lines, one dedicated solely to the carrier, and a second one merged into a bubbler through which the analyte flow was generated. The two lines recombined the flow immediately before the testing chamber inlet so that different concentrations of the analytes were produced by controlling the relative flow of the two lines. The total flow delivered in the testing chamber was kept at a constant rate of $1000 \mathrm{sccm}$. The sensors were refreshed by solely flowing argon inside the testing chamber at the end of each analyte delivery phase. A complete testing cycle was hence formed by sequencing both analyte and refresh phases of identical duration (285 seconds each). A Labview controlled system of two and three-way solenoid valves switched the flows so that the two phases were automatically alternated.

The die under test was attached to a custom designed PCB that is provided with on-board calibration standards and served as a lid for the testing chamber. The resonators were wirebonded to $50 \Omega$ lines and the analytes-induced frequency shift were monitored by an Agilent ${ }^{\circledR}$ N5230A Network Analyzer connected to the PCB.
As mentioned above, the effect of two distinct single strands of DNA as functionalizing agents for SWNTs have been investigated. Analyte concentrations equal to 10,25 and $50 \%$ of the saturated vapor pressure were delivered on both sensors.

In order to observe the effect of the nanofunctionalization layer, the sensor response was characterized for the same analytes with just bare SWNTs and after the application of ss-DNA .

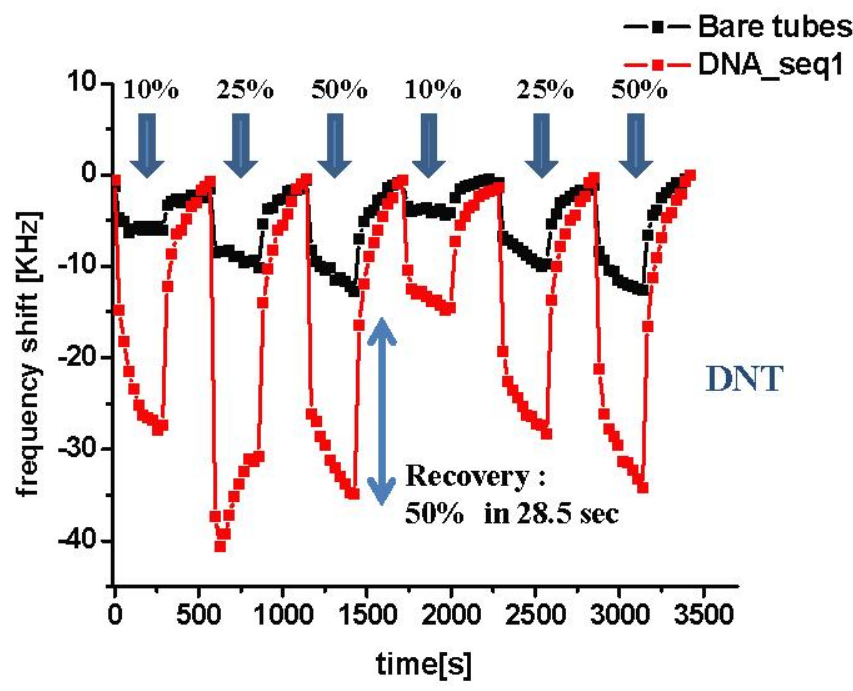

Figure 4: Response to DNT of a $450 \mathrm{MHz}$ AlN contourmode resonator with and without DNA seq.1 functionalization. The sensor is refreshed to its original state by flowing Ar. No external heating is required and $50 \%$ recovery is achieved in 28.5 seconds.

Figure 4 shows the frequency shift that was recorded for the $450 \mathrm{MHz}$ resonator after the application of ssDNA sequence 1. The data demonstrate a net enhancement in the adsorption of DNT with respect to bare SWNTs. As for DMMP, an adsorption enhancement was also recorded, but it was just a factor of 2-3 X compared to the 8 fold increase obtained for DNT.

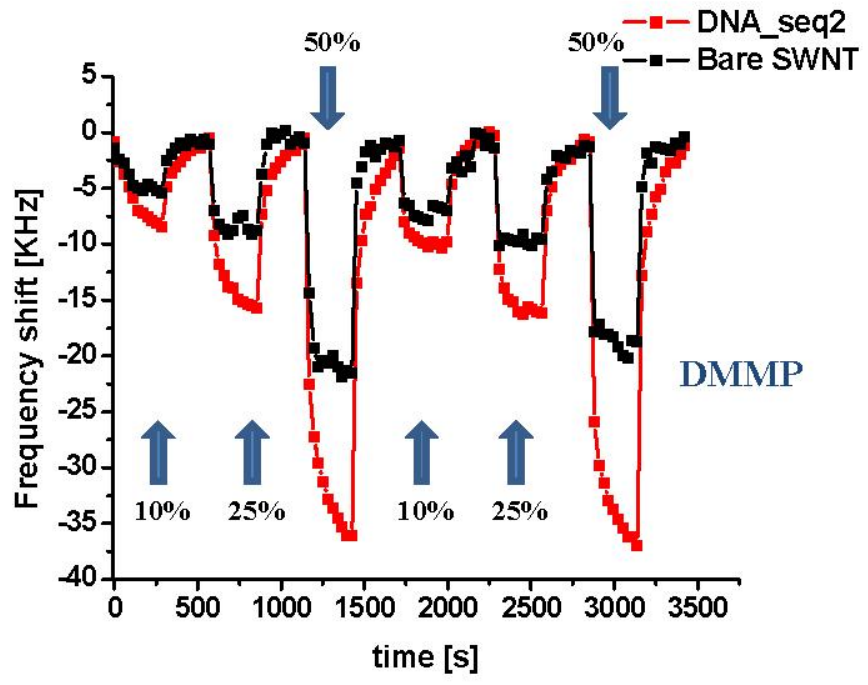

Figure 5: Response to DMMP of a $287 \mathrm{MHz}$ AlN Contour-Mode resonator with and without DNA seq. 2 functionalization 
The ss-DNA nano-enabled resonant sensor at $287 \mathrm{MHz}$ and functionalized with sequence 2 exhibited a clear improvement in the response to both chemical agents. Differently, in this case, no substantial diversification in the response to DNT and DMMP was recorded; in fact, both species adsorption was amplified by a factor of nearly 2-3 folds over the bare SWNT-coated resonator

During the refresh phase both sensor responses recovered at least $50 \%$ of their initial value in approximately 28.5 seconds, demonstrating that the sensor is reversible.

Although the adsorption mechanism is not fully understood and further investigations are needed, from the previous results it can be inferred that the DNA nanocoating layer increases the binding affinity between the analyte and the SWNT adsorbent layer, therefore enhancing the amount of adsorbed mass for a given concentration.

The amount of mass adsorbed on the nano-sensitive layer for each analyte concentration has been estimated from the change in the motional inductance of the extracted MBVD circuit (Fig.6). The adsorbed mass, $\Delta m$, is modeled by a series motional inductance, $\mathrm{Lm}^{*}$, proportional to the adsorbed mass and a series resistance, $R m^{*}$, that takes into consideration the small additional damping introduced by the adsorption process:

$$
\Delta m=2 \cdot n \cdot L m^{*} \cdot \eta^{2}
$$

where $\eta$ is the electromechanical coupling coefficient of the resonator $\left(\eta=2 \cdot d_{31} E_{o} L\right)$.

The estimated VOC mass adsorbed for $10 \%$ concentration of both analytes is reported in Figure 7 , where the diversification in the affinity to different chemical species clearly points out that the adsorption process is DNA sequence dependent.

After the application of the DNA solution, a decrease in $Q$ factor of approximately $10 \%$ (considering the value of $Q$ after flowing Ar) was recorded for both devices. The partially aqueous environment created by the addition of DNA is considered the primary cause of this additional damping. No change in the off-resonance capacitance was observed, while $R m$ slightly increased (approximately $10 \%$ of its original value) reflecting the corresponding drop in $Q$.

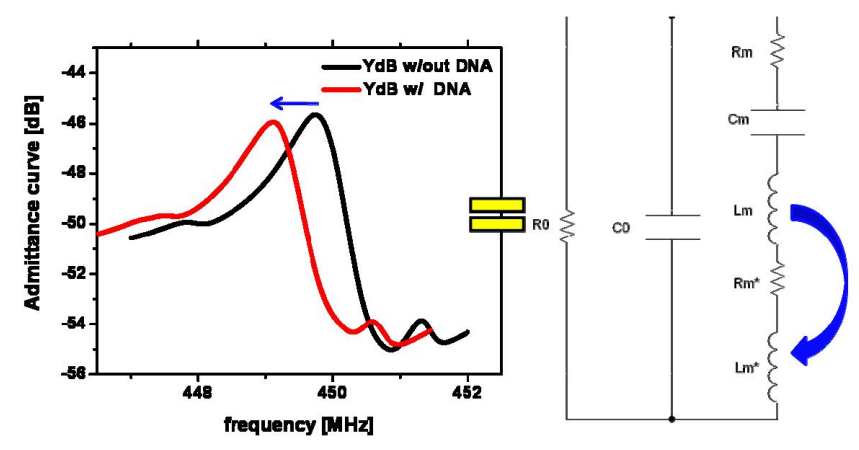

Figure 6: The admittance curve of the resonant device was measured before and after the DNA deposition and both curves were fitted to the Modified Butteworth van Dyke (MBVD) model to extract the added mass of DNA on the resonator surface. A total DNA mass of $230 \mathrm{pg}$ was attached to the resonator. The same technique was employed to extract the added mass due to adsorption of $V O C$.

\section{CONCLUSION}

A novel nano-enabled gravimetric chemical sensor prototype based on Ss-DNA decorated SWNTs on AlN contour-mode MEMS resonator has been designed, fabricated and tested.

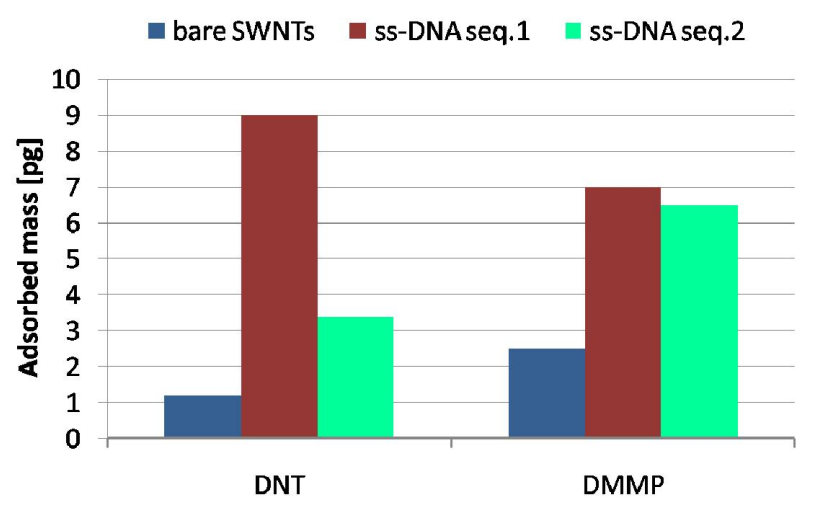

Figure 7: Comparison between DNT and DMMP response with different ss-DNA functionalization sequences for $10 \%$ analyte concentration. The chart shows the enhanced mass adsorption occurred because of the functionalization process and the ability to selectively distinguish between DNT and DMMP.

The novel functionalization layer permits to achieve up to an 8 fold increase in sensitivity to DNT over bare nanotubes with 1 sequence of DNA, and a 2-3 fold enhancement to both DNT and DMMP when a second sequence is employed. This work constitutes the first experimental verification towards the demonstration of a highly selective nanomechanical nose, whose realization will be made possible by gaining access to a vast ss-DNA library.

\section{REFERENCES}

[1] T. Zhang, S. Mubeen, N. V. Myung, M. Deshusses, "Recent progress in carbon nanotube-based gas sensors", Nanotechnology 19, 2008.

[2] M. Rinaldi, C. Zuniga et al. "Gravimetric chemical sensor based on the direct integration of SWNTs on AIN contour-mode MEMS resonators", IEEE IFCS 2008 proceedings, pp. 443-448.

[3] A. Star, T. R. Han, V. Joshi, J. C. Gabriel, and G. Gruner, Adv. Mater. 16, 2004.

[4] K. Besteman et al. Nano Lett., vol. 3, No.6, pp. 72730, 2003

[5] M. Penza et al., Appl. Phys. Lett. 90, 173123, 2007.

[6] C. Staii, A. T. Johnson, M. Chen, A. Gelperin, Nano Lett., vol. 5 No. 9, pp. 1774-1778.

[7] N.R. Franklin, Y. Li, R.J. Chen, "Patterned growth of single walled carbon nanotubes on a full 4-inch wafer", Appl. Phys. Lett. 79, No. 27, 2001.

[8] M. Rinaldi, C. Zuniga and G. Piazza, "5-10 GHz AlN contour mode Nanoelectromechanical MEMS resonators" , IEEE MEMS 2009 proceedings.

[9] G. Piazza, P. J. Stephanou, A. P. Pisano, J. of Microel. Syst., Vol.15, Dec.2006, pp1406-1418. 